\title{
Influence of selecting EPI readout-encoding bandwidths on arterial spin labeling perfusion MRI
}

\author{
Geon-Ho Jahng • Norbert Schuff
}

Received: 4 February 2009 / Revised: 10 June 2009 / Accepted: 11 June 2009 / Published online: 4 July 2009

(c) The Author(s) 2009. This article is published with open access at Springerlink.com

\begin{abstract}
Object The objective of this study was to investigate effects of varying readout bandwidths on the arterial spin labeling (ASL)-perfusion MRI measurements at a high magnetic field MRI system.

Materials and methods Brain perfusion studies were performed on nine volunteers (four males, five females) using flow sensitive alternating inversion recovery (FAIR) ASL single-shot echo-planar imaging (EPI)-MRI. To investigate EPI bandwidth effects on the time-series perfusion-weighted imaging (PWI) data, two regions-of-interest (ROI) were placed outside the brain to determine the level of noise and another ROI inside the brain to determine the level of signal. Coefficients of variations $(\mathrm{CoV})$ were calculated for the timeseries PWI data. One-way analysis of variance (ANOVA) was used to investigate voxel-wise differences in the time-series PWI data between two different bandwidth values.

Results At the level of ROI, there was no significant effect of changing EPI bandwidths on the time-series PWI data in any of the volunteers $(P>0.031)$. In contrast, $\mathrm{CoV}$ values over the dynamic PWI data varied with depending on selecting EPI bandwidths and voxel-based tests showed that N2 ghosting, modulated by EPI bandwidth, can appear in some brain regions, especially in areas that overlap with the spatial distribution of N2 ghosting artifacts.
\end{abstract}

\section{G.-H. Jahng ( $\varangle)$}

Department of Radiology, School of Medicine,

East-West Neo Medical Center, Kyung Hee University,

149 Sangil-dong, Gangdong-gu, Seoul 134-090, South Korea

e-mail: ghjahng@gmail.com

\section{N. Schuff}

Department of Radiology, Center for Imaging of Neurodegenerative Diseases, VA Medical Center, University of California, San Francisco, 4150 Clement Street, 114M, San Francisco, CA 94121, USA
Conclusions Although N2 ghosting can be reduced by adjusting the bandwidth of EPI on the time-series of PWI data, the effects cannot be entirely eliminated. In particular, $\mathrm{N} 2$ ghosting can bias CBF quantification if EPI control scans to determine the equilibrium-state signal are confounded by N2 ghosting. Therefore, careful tuning of the bandwidth of EPI is necessary to avoid artifacts in the ASL signal from N2-ghosting.

Keywords Nyquist ghost artifact · Echo-planar imaging · Readout-encoding bandwidth · Arterial spin labeling . Human brain $\cdot$ High field MRI

\section{Introduction}

Echo-planar imaging (EPI) [1,2], which facilitates a speedy acquisition of the magnetic resonance signal, is nowadays widely used for generating maps of various signal contrasts, such as the blood oxygenation level dependent (BOLD) contrast in functional MRI (fMRI), diffusion tensor imaging (DTI), and cerebral blood flow (CBF) using arterial spin labeling (ASL) MRI. However, in addition to factors of eddy current, echo misalignment, or gradient/receiver miscalibration, the rapidly switching magnetic field gradients of EPI can result in considerable mechanical vibrations which lead in turn to magnetic field fluctuations causing Nyquist (N2) ghosting in the EPI data [1-3]. Although many strategies have been invented to reduce $\mathrm{N} 2$ ghosts, including gradient compensation [4], timing corrections [5], reference phase corrections [6], and post-processing image restoration [7], none of these methods can entirely eliminate this artifact. Moreover, the corrections may not be effective for a time variant signal, such as the ASL or BOLD signal, because 
temporal variations of the signal and the gradient vibrations are usually not synchronized.

Perfusion MRI based on the ASL technique is generally performed using a gradient-echo EPI sequence to map CBF $[8,9]$. EPI provides the highest signal-to-noise ratio (SNR) for a given repetition time compared to other sequences. The flow sensitive alternating inversion recovery (FAIR) ASLMRI method is one of several techniques to label arterial blood water [10]. In FAIR, a control image (unlabeled image) is acquired with a slice selective inversion recovery pulse and a labeled image is acquired with a non-slice-selective inversion recovery pulse. A perfusion-weighted image (PWI) is obtained by subtracting the labeled image from the control image. The values of PWI can be transformed in principle into absolute units of using kinetic models of blood flow [11].

Recently, the author [12] and others [13] demonstrated for DTI that the impact of N2 ghosting on diffusion measures can practically be eliminated by tuning the modulation frequency of the EPI readout gradient, i.e. the EPI bandwidth, to a range outside the harmonics of mechanical gradient vibrations so that the magnitude of the vibrations is substantially reduced. The objective of this study was to investigate whether the same strategy in reducing mechanical gradient vibrations is also effective in eliminating N2 ghosting in ASL-MRI measurements that are involved in the temporal variations of the signals. Specifically, we tested the extent to which residual N2 ghosting, induced by EPI gradients, can corrupt timeseries measurements of PWI.

\section{Materials and methods}

The local institutional review boards of the VA medical center and the University of California approved this study. Informed consent was obtained from all participants prior to enrolling in this study. Nine volunteers (four males, five females) were recruited from the local community. $\mathrm{T}_{1}$-weighted and $\mathrm{T}_{2}$-weighted structural images and ASL-MRI data were obtained for each volunteer using a 4 Tesla MRI system (Bruker/Siemens MedSpec, Germany) equipped with a birdcage RF transmit head coil and an eight-channel receiver head coil.

\section{Data acquisitions}

For the human brain study, the FAIR-ASL [10] method was used for spin labeling to obtain control (i.e. unlabeled) and labeled EPI images with ascending acquisitions. FAIR-ASL was combined with QUIPSS II with thin-section $\mathrm{TI}_{1}$ periodic saturation (Q2TIPS) gradient-echo EPI sequence [14] to define the length of the ASL bolus, improving measure- ment accuracy. The in-plane inversion slab thickness was $150 \mathrm{~mm}$ for the slab-selective inversion pulse. The post-labeling delay time $\left(\mathrm{TI}_{1}\right)$ between the inversion pulse and the start of the periodic saturation pulse was $900 \mathrm{~ms}$ and the labeling delay time $\left(\mathrm{TI}_{2}\right)$ between the inversion and the EPI excitation pulse was $1,900 \mathrm{~ms}$. The acquisition matrix of EPI was $48 \times 64$ with $6 / 8$ phase partial Fourier acquisitions along the phase direction of EPI to shorten geometrical distortions in EPI. The repetition time (TR) and echo time (TE) were $3 \mathrm{~s}$ and $9 \mathrm{~ms}$, respectively and they was kept constants in all experiments. The field-of-view (FOV) was $240 \times 256 \mathrm{~mm}^{2}$ without using a phase array reconstruction. Eleven transversal slices were acquired with $6 \mathrm{~mm}$ slice thicknesses and a $2 \mathrm{~mm}$ gap between slices to cover brain regions. Eighty ASL measurements were averaged to obtain time-dependent PWI signal changes, yielding the acquisition time of four minutes. A 3D online motion correction program during scans provided by the manufacturer was used to minimize motion artifacts. To cover a range of $\mathrm{N} 2$ ghosting, the EPI readout-encoding bandwidths of 2.442 (the echo-spacing time, $\mathrm{ES}=0.47 \mathrm{~ms}$, yielding strong N2 ghosting), 2.790 ( $\mathrm{ES}=0.42 \mathrm{~ms}$, weak N2 ghosting), to $3.004(\mathrm{ES}=0.40 \mathrm{~ms}$, another weak N2 ghosting) $\mathrm{kHz} /$ pixel were selected based on the following phantom study. Please note that ES is the time between two neighboring EPI echoes.

Phantom studies were performed using a spherical water phantom provided by the manufacturer to determine N2 ghosting as a function of EPI bandwidth values. A gradientecho EPI sequence was used with the following parameters: the matrix size of $64 \times 64$, five transversal slices each $5 \mathrm{~mm}$ thick $10 \mathrm{~mm}$ apart, and a nominal voxel size of $4 \times 4 \times 5 \mathrm{~mm}^{3}$ with $\mathrm{FOV}=256 \times 256 \mathrm{~mm}^{2}$. TR and TE were $3 \mathrm{~s}$ and $30 \mathrm{~ms}$, respectively with four averages taken. The phase encoding gradient of EPI was oriented along the anterior-posterior direction. The bandwidth of the EPI readout-encodings was varied from $1.346 \mathrm{kHz} /$ pixel (i.e. $86.144 \mathrm{kHz}$, corresponding to $\mathrm{ES}=0.81 \mathrm{~ms}$ ) to $3.720 \mathrm{kHz} /$ pixel (i.e. $238 \mathrm{kHz}$, corresponding to $\mathrm{ES}=0.39 \mathrm{~ms}$ ). Regions of interest (ROI) were drawn in an area of N2 ghosting in the unlabeled raw EPI images as showing in Fig. $1 \mathrm{a}$ and the mean value of the ghost intensity computed. Intensity variations of N2 ghosting as a function of the EPI readout bandwidth are shown in Fig. 1b. In addition, to repeat the phantom experiments under FAIR ASL-MRI conditions, five transversal slices were acquired with the $6 \mathrm{~mm}$ slice thickness and $6 \mathrm{~mm}$ apart between slices to minimize slice cross-talk effect of imperfection of the slice-selection pulses and four measurements were averaged. The EPI readout-encoding bandwidth was varied from $2.442 \mathrm{kHz} /$ pixel (corresponding to $\mathrm{ES}=$ $0.47 \mathrm{~ms}$ ) to $3.552 \mathrm{kHz} / \mathrm{pixel}$ (corresponding to $\mathrm{ES}=0.38 \mathrm{~ms}$ ). Except for the number of slice and averages, the rest of imaging parameters were identical to the ASL-MRI scans of humans. 

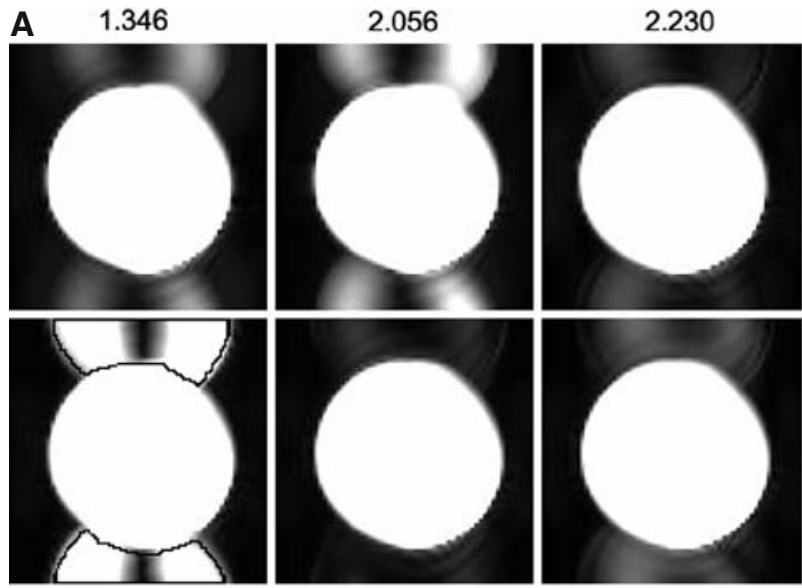

2.442

3.004

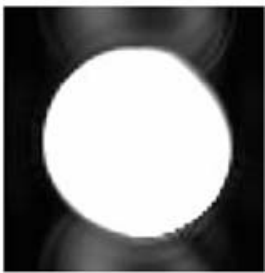

3.552

B
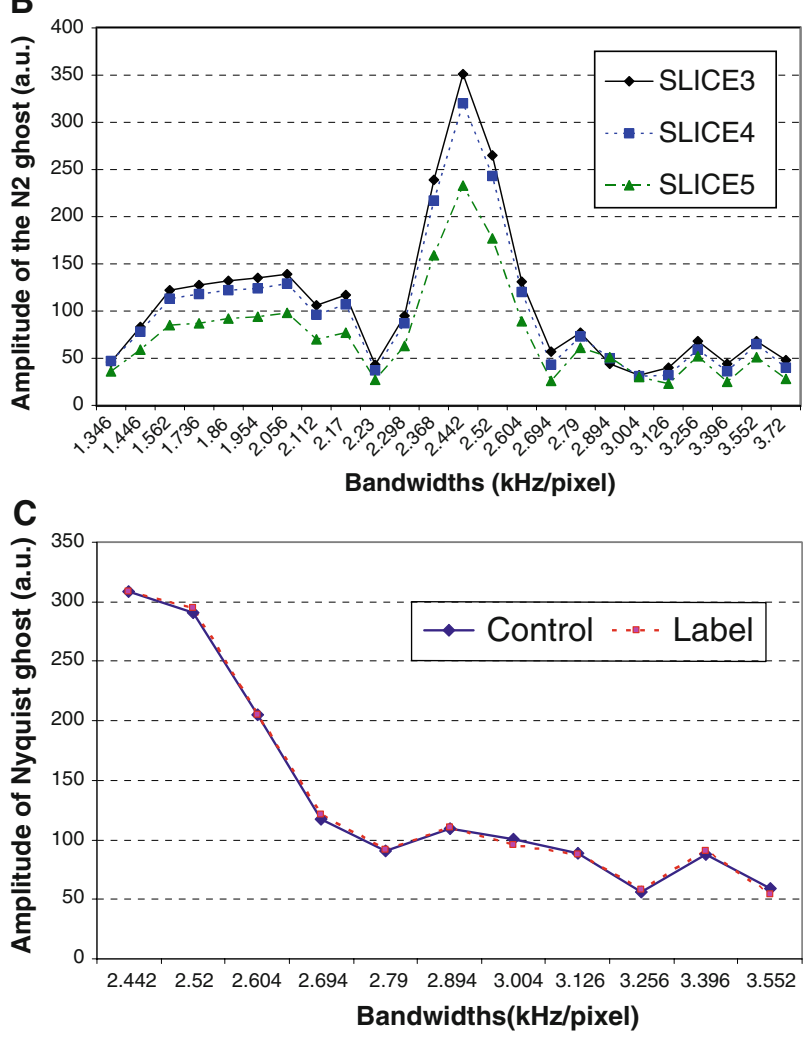

Fig. 1 Representative images of the phantom scans (Fig. 1a) from the third slice for a few EPI readout-encoding bandwidths $(\mathrm{kHz} / \mathrm{pixel})$ and the Nyquist (N2) ghost behaviors on matrix of $64 \times 64 \mathbf{b}$ and on FAIR ASL MRI condition $\mathbf{c}$ of the gradient-echo EPI sequence. In a, the window brightness is set the same for all six images to emphasize the $\mathrm{N} 2$ ghost. The amplitudes of $\mathrm{N} 2$ ghosting on three slices are shown in $\mathbf{b}$ that data are obtained from the ROI in $\mathbf{a}$. Three readout-encoding bandwidths for further applications on the human brain are selected based on the results of the phantom studies

\section{Imaging processing}

For the brain data analyses, Statistical Parametric Mapping Version 2 (SPM2) software (http://www.fil.ion.ucl.ac. $\mathrm{uk} / \mathrm{spm} / \mathrm{software} / \mathrm{spm} 2 /$ ) was used for data post-processing

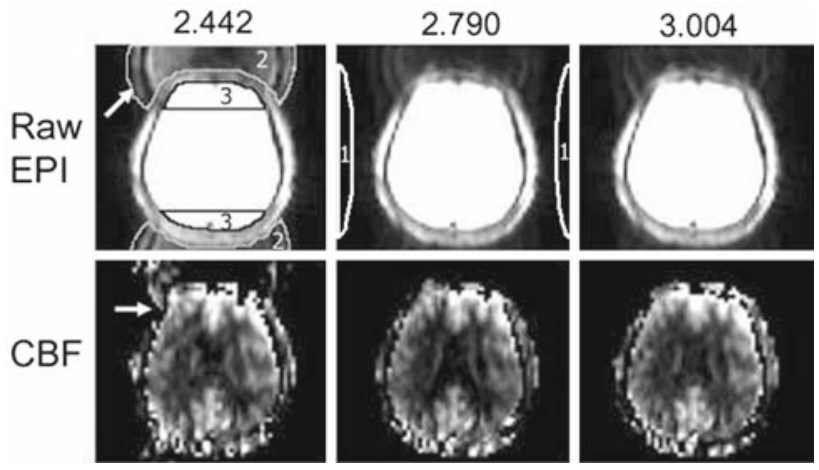

Fig. 2 Representative images and results of the arterial spin labeling (ASL)-MRI human brain study. Representative images of the averaged gradient-echo EPI images of control and labeled scans (Raw EPI) and the corresponding cerebral blood flow (CBF) maps are shown in Fig. 2. In Fig. 2, the window brightness is set the same for three EPI images to emphasize the Nyquist (N2) ghost. Similar to the phantom study, three areas of regions-of-interest (ROI) are defined for each bandwidth and labeled as 1(the right-left areas outside of brain), 2(the anterior-posterior areas outside of brain), and 3 (inside of brain)

and evaluations. For each EPI readout-encoding bandwidth, the motion corrected 80 dynamic EPI images and an averaged image of the motion corrected images was created from the original 80 images of the FAIR ASL-MRI data. Time-series PWI data processed by subtracting between the control images and the labeled images were created using a Matlab script. Control images, labeled images, and PWI data for each bandwidth were spatially normalized to the EPI.mnc template of SPM2. This accomplished an analysis by voxel-wise as well as by ROI at consistent anatomical locations.

To calculate averaged CBF maps over 80 subtracted images for each volunteer, shown in Fig. 2, the PWI sets were scaled by using an in-house built program based on a single component and instantaneous exchanging model for ASL [11] according to:

$\Delta M=2 M_{0 t} \cdot f \cdot \frac{\alpha}{\lambda} \cdot T I_{1} \cdot e^{\left(-T I_{2} / T_{1 b}\right)} \cdot E$

Here, $\Delta M$ is the voxel-based subtracted image, $M_{o t}$ is the control scan signal, $f$ indicates CBF in units of $\mathrm{ml} / 100 \mathrm{~g}$ per minute. The factors $\alpha=0.98$ and $\lambda=1.0 \mathrm{ml} / 100 \mathrm{~g}$ describe the labeling efficiency and the tissue-to-blood partition, respectively. $\mathrm{TI}_{1}$ and $\mathrm{TI}_{2}$ are 900 and $1,900 \mathrm{~ms}$, respectively. $\mathrm{TI}_{2}$ is also a function of the slice order as blood flow advances from the labeling gap to the distal slice position in the brain. Hence, the imaging time of EPI is added cumulatively to $\mathrm{TI}_{2}$ for each ascending slice. $T_{1 b}=1,620 \mathrm{~ms}$ is the longitudinal relaxation time of blood [15]. $E=1$ is a correction factor to account for potential scaling of longitudinal relaxation times of brain tissue $\left(T_{1 t}\right)$ and blood $\left(T_{1 b}\right)$. 


\section{Statistical tests}

For the ROI-based analysis of brain PWI data, three ROIs were drawn for each bandwidth and were evaluated to determine the time-dependent variations of the $\mathrm{N} 2$ ghost on the ASL-MRI data. One ROI (ROI1) was placed outside of the brain and distant from regions with potential N2 ghosting, as shown in Fig. 2. Another ROI (ROI2) was placed again outside of the brain but in a region with $\mathrm{N} 2$ ghosting. ROI1 was select to measure changes in the noise level as EPI bandwidth was changed and ROI2 was selected to measure the intensity of N2 ghosts. Finally, to determine the ASL signal level, a third ROI (ROI3) was placed either in anterior and posterior brain areas that overlapped with N2 ghosting, as shown in Fig.2. For each time-series ROI data, variations in the ROIs between different bandwidth values were evaluated using two-way $T$ tests, separately for each volunteer. To account for multiple comparisons from the three bandwidth tests, we adjusted the level of significance to $\alpha=0.016(0.05 / 3)$. In addition, to compare the effect of bandwidth with variations within subjects, a linear mixed effects model was designed to separate out random variations within subjects from fixed effects related to bandwidth.

For the voxel-based analyses, we first smoothed the normalized PWI data with an Gaussian kennel of $8 \mathrm{~mm} \times 8 \mathrm{~mm} \times$ $10 \mathrm{~mm}$ and then tested for effects of bandwidth using the general linear model framework in SPM2. One-way analysis of variance (ANOVA) was used to investigate the voxel-wise differences of the time-series PWI data between two different bandwidths for each subject. The statistically significant level for each comparison was $\alpha=0.005$ without further corrections of multiple comparisons ( $P=0.005$, none).

\section{Results}

Representative images of the water phantom acquired to the $64 \times 64$ imaging matrix with the gradient-echo EPI sequence are shown in Fig. 1a for several bandwidths of EPI readout-encoding. The window brightness is kept the same for all six images to emphasize the change in magnitude of the N2 ghosts for the different EPI readout-encoding bandwidths. This shows the magnitude of the N2 ghost varied substantially over the range of bandwidth values. Using the ROI shown in Fig. 1a, we obtained the amplitudes of the N2 ghost on the three middle slices shown in Fig. 1b. ANOVA showed significant effects on both EPI readout-encoding bandwidths $(F=65.16, P<0.0000001)$ and imaging slices $(F=35.45, P<0.00001)$. Although the amplitude of the N2 ghost had similar behaviors for the three slices, there were significant differences between slice five and three or five and four $(P<0.0001)$, but there was no significant difference between slice three and four $(P>0.05)$. The highest
Table 1 Summaries of the human brain study for the averaged PWI (subtracted) data with the FAIR-arterial spin labeling MRI obtained from nine volunteers

\begin{tabular}{llll}
\hline Bandwidths (kHz) & nPWI (ROI1) & nPWI (ROI2*) & nPWI (ROI3) \\
\hline 2.442 & $0.050 \pm 0.398$ & $1.198 \pm 3.850$ & $7.143 \pm 16.879$ \\
2.790 & $0.045 \pm 0.268$ & $0.468 \pm 1.124$ & $7.082 \pm 16.097$ \\
3.004 & $0.040 \pm 0.203$ & $0.429 \pm 1.778$ & $7.259 \pm 13.573$
\end{tabular}

The normalized means and standard deviations over all subjects are listed for the three regions-of-interest (ROI) and the three readout-encoding bandwidths defined in the Fig. 2. The PWI ROI values are normalized by using those from the brain ROI (ROI3) of averaged raw EPI data (i.e. BOLD) for each bandwidth and for each volunteer $(\mathrm{nPWI}$ value $=1,000 \times \mathrm{PWI} / \mathrm{BOLD}$ in ROI3)

$* P<0.000001$ : significant differences between $\mathrm{BW}=2442$ and $\mathrm{BW}=2,790 / \mathrm{BW}=3,004$, EPI: echo planar imaging, FAIR: flowalternated inversion recovery, nPWI: normalized PWI

amplitude of the $\mathrm{N} 2$ ghost was obtained at the EPI readoutencoding bandwidth of $2.442 \mathrm{kHz} /$ pixel, corresponding to an ES of $0.47 \mathrm{~ms}$.

Figure 1c shows variations in N2 ghosting as a function of bandwidth, separately for the control and the labeling image for the phantom. Adding to EPI the ASL preparation part had no effect on N2 ghosting $(F=0.176, P=0.677)$ as expected since the bandwidth values for scans with and without ASL preparation were the same. The ANOVA test also showed no effects by different acquisitions (two acquisitions for averaging) $(F=0.540, P=0.467)$, implying that the EPI gradients are stable during multiple acquisitions. However, there were significant readout-encoding bandwidth effects ( $F=3909.3, P<0.000001)$. Within the ROI, the amplitudes of the N2 ghost on the averaged images of the control and the labeled scans are dependent on the selection of the EPI readout-encoding bandwidths.

Results of the studies on nine volunteers are listed in Table 1, separately for the three ROIs and the three EPI bandwidth values. The normalized means and standard deviations over all subjects are computed based on the three ROIs and the three readout-encoding bandwidths defined in the Fig. 2. The PWI ROI values are normalized by using those from the brain ROI (ROI3) of averaged raw EPI data (i.e. BOLD) for each bandwidth (BW) and for each volunteer, according to $\mathrm{nPWI}$ value $=1,000 \times \mathrm{PWI} / \mathrm{BOLD}$ in ROI3. Values in ROI2 at $2.442 \mathrm{kHz} /$ pixel are significantly different compared to those at $2.790 \mathrm{kHz} /$ pixel $(P<0.000001)$ or at $3.004 \mathrm{kHz} /$ pixel $(P<0.000001)$. In contrast to ROI2, variations in the other two ROIs, in particular in RO3 located in the brain, were not significant. Table 1 also demonstrates that the $\mathrm{N} 2$ ghost amplitude in ROI2 is strongly depended on the selections of the bandwidth, but this dependence is diminished in the brain ROI.

Figure 2 shows the representative EPI images of the averaged gradient-echo EPI images of control and labeling scans and the corresponding CBF maps obtained from a volunteer 

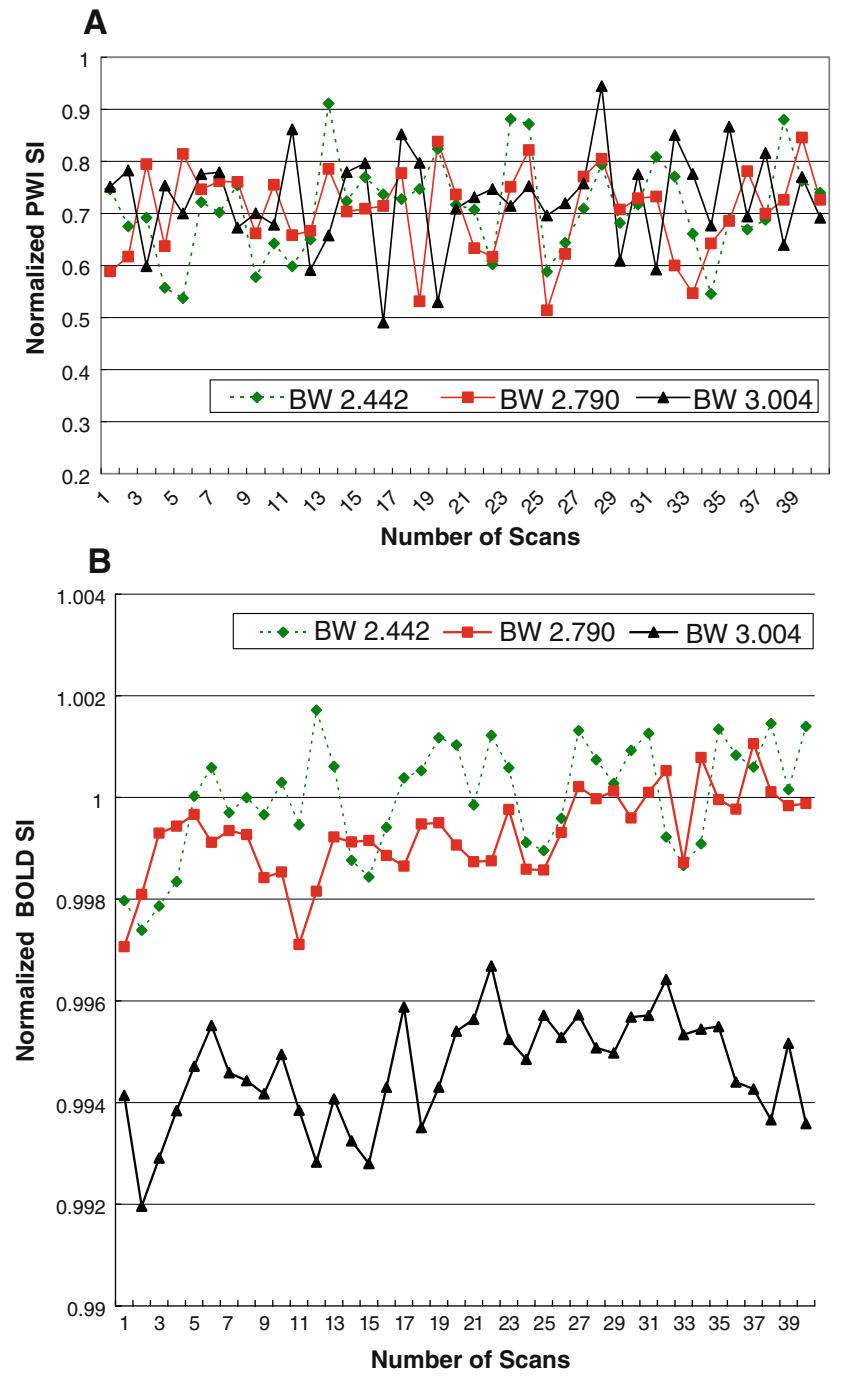

Fig. 3 The normalized time-series PWI data (subtraction, a) and the corresponding averaged EPI data (addition or BOLD, b) from three different EPI bandwidths obtained from the brain ROI for all volunteers. The BOLD signal is normalized to the mean of all time points. SI signal intensity

using the FAIR ASL-MRI. The ROIs without (region 1) and with (region 2) N2 ghosting outside the brain (background) and one ROI (region 3) inside the brain regions to represent signal intensities are highlighted. As pointed out in Table 1 , intensity variations in ROI2 are significantly different between 2.442 and $2.790 \mathrm{kHz} /$ pixel $(P<0.000001)$ or $3.004 \mathrm{kHz} /$ pixel $(P<0.000001)$ in ROI2, but there is no significant difference between the other EPI bandwidths for the ROI2. In addition, the other ROIs show no significant dependence from bandwidth. N2 ghosting is dominant at $\mathrm{BW}=2.442 \mathrm{kHz} /$ pixel $(\mathrm{ES}=0.47 \mathrm{~ms})$, less dominant both at $\mathrm{BW}=2.790 \mathrm{kHz} /$ pixel $(\mathrm{ES}=0.42 \mathrm{~ms})$ and at $\mathrm{BW}=3.004$ $\mathrm{kHz} /$ pixel $(\mathrm{ES}=0.4 \mathrm{~ms})$, consistent with the result of the phantom study. N2 ghosting inside the brain areas is indicated on the CBF map shown in Fig. 2 by the white arrow.
Table 2 Means and standard deviations of cerebral blood flow (CBF) values of the two ROIs shown in the Fig. 2 for the three EPI readoutencoding bandwidths over all subjects

\begin{tabular}{lcl}
\hline Bandwidths $(\mathrm{kHz})$ & \multicolumn{2}{c}{$\mathrm{CBF}(\mathrm{ml} / 100 \mathrm{~g}$ per minute $)$} \\
\cline { 2 - 3 } & $\mathrm{N} 2$ noise ROI2 & Inside brain ROI3 \\
\hline 2.442 & $19.03 \pm 8.13$ & $79.67 \pm 7.03$ \\
2.790 & $2.13 \pm 2.35$ & $78.53 \pm 5.63$ \\
3.004 & $2.21 \pm 2.45$ & $79.43 \pm 5.85$ \\
\hline
\end{tabular}

${ }^{\mathrm{a}}$ Based on the ANOVA test (dependent variable $=$ ROI values, independent variable $=$ bandwidth), amplitudes of the Nyquist (N2)-noise ROI region are significantly associated with variations of EPI readout-encoding bandwidths on CBF maps $(F=32.86, P<0.00001)$. The Scheffe post hoc test shows that there are significant differences between at $2.442 \mathrm{kHz} /$ pixel and at $2.79 \mathrm{kHz} /$ pixel bandwidth or at $3.004 \mathrm{kHz} /$ pixel bandwidth on the CBF map $(P<0.0001)$

Figure 3 shows the normalized time-series PWI data (Fig. 3a) and the corresponding averaged EPI data (addition or BOLD, Fig. 3b) obtained from the brain ROI of all volunteers. The time-series data are normalized to average value of control or unlabeled values obtained from the brain ROI for each volunteers. The BOLD signal is normalized to the mean of all time points. CoV values of the normalized PWI data over the dynamic scans are 47, 34.78 and $32.92 \%$ for $2.442,2.790$, and $3.004 \mathrm{kHz} /$ pixel, respectively. Using the ROI data, we also compared the time-series PWI data for the three EPI bandwidth values, separately for each volunteer. With an adjusted significant level of $\alpha=0.016$ $(0.05 / 3$ tests), there are no significant difference among any EPI bandwidths for the time-series PWI data for any volunteer $(P>0.031)$. Variations may have been attenuated by averaging over relatively large ROIs. Therefore, we performed voxel-based analysis. The result using a linear mixed effect model showed statistically significant differences for ROI2 $(P<0.0001)$ but not for ROI1 and ROI3 $(P>0.05)$.

Table 2 lists CBF values for each EPI readout-encoding bandwidth obtained from ROI2 (outside the brain) and ROI3 (inside brain) shown in the Fig. 2. The CBF values are in units of $\mathrm{ml} / 100 \mathrm{~g}$ per minute. Using multiple factor ANOVA with CBF values in the ROI as dependent and bandwidths as independent variable, the result showed the marked effect on CBF measurements in ROI2 as a functional of EPI readoutencoding bandwidths $(F=32.86, P<0.00001)$, whereas variations in ROI3 region were not significant $(F=0.084$, $P=0.919)$. Post hoc test showed that the marked CBF variations in ROI2 were primarily due to differences between the low bandwidth at $2.442 \mathrm{kHz} /$ pixel relative to the intermediate bandwidth at $2.79 \mathrm{kHz} /$ pixel as well as to the high bandwidth at $3.004 \mathrm{kHz} /$ pixel $(P<0.0001)$, but difference between intermediate and high bandwidth was not significant $(P>0.15)$. 


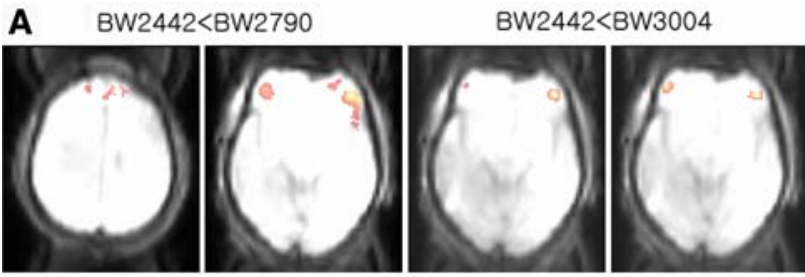

B

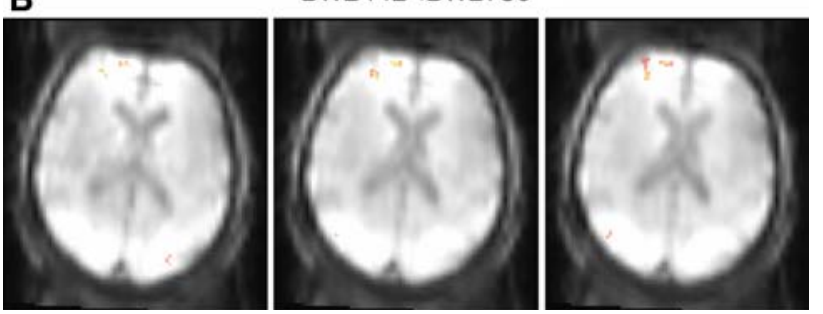

Fig. 4 The results of the voxel-based analyses of the time-series PWI data obtained from two different volunteers $(\mathbf{a}, \mathbf{b})$. The sign " $<$ " means statistical comparisons between two data. In the figure, we adjusted the imaging contrast to emphasize $\mathrm{N} 2$ ghost artifacts outside brain

Results of the voxel-based analyses of the time-series PWI data are depicted in Fig. 4 for two different volunteers ( $4 \mathrm{a}$ and $4 \mathrm{~b}$ ). In Fig. $4 \mathrm{a}$, the time-series PWI data vary between low and intermediate or high bandwidth values in some voxels, indicating the influence of N2 ghost, especially the map of the most left image. In Fig. 4b, similar effects also can be found in another subject, although in different slices. Timeseries PWI data also differ between low and intermediate bandwidth in some voxels in the frontal and occipital lobes that might be explained by $\mathrm{N} 2$ ghost. In Fig. 4, we adjusted imaging contrast to emphasize N2 ghost artifacts outside brain. For the time-series raw EPI data (average of unlabeled and labeled images for each time-point), we found significant differences between the bandwidth values for all subjects in whole brain for any comparisons, as expected

\section{Discussion}

The major finding of this study is that N2 ghosting can have a significant effect on the ASL signal in some regions, as shown in a voxel-based analysis. This is, in principle, possible, because the mechanical gradient vibrations may induce a spatially non-uniform fluctuating magnetic field. Another explanation, however, is that the artifacts are due to susceptibility variations between the acquisitions. However, the highlighted area of the most left map in Fig. 4a is likely not an artifact of susceptibility because the slice position is relatively distant from regions with known high susceptibility distortions and further the subject was at rest and did not perform any functional tasks during the measurement. Despite the conservative threshold value used $(P=0.005)$ for voxelbased comparisons, it could also be possible that these effects occurred just by chance given the large number of voxels and tests.

Although PWI data are sometimes used in evaluations of ASL-MRI data, quantitative CBF calculation can be a benefit for many physiological or clinical studies. Quantitative CBF measurements usually require information of $M_{0 b}$, which is the equilibrium-state signal of blood obtained from an EPI data in full relaxation condition. Researchers often use the control EPI image $\left(M_{0 t}\right)$ to approximate $M_{0 b}$ or measure $M_{0 b}$ images using a single-shot EPI with an infinite value for TR. The assumption $M_{0 b}=M_{0 t}$ can then lead to an error if $M_{0 t}$ involves $\mathrm{N} 2$ ghosting when an inappropriate bandwidth is used. This can even be true for both the control and the labeled images. Although subtracting the labeled images from the control images $(\Delta \mathrm{M})$ may diminish $\mathrm{N} 2$ ghosting, the effect is re-introduced by $\mathrm{CBF}$ quantification through the computation $\Delta M / M_{0 t}$. In addition, errors of CBF quantifications due to using $M_{0 t}$ to represent $M_{0 b}$ can be as high as 11.3 and $18.7 \%$ in gray matter and white matter, respectively in human brain.

We found no significant effect of N2 ghosting in PWI data based on the ROI analysis. One explanation for this result is that subtractions of labeled from unlabeled ASL data may reduce the effect of $\mathrm{N} 2$ ghosting on the perfusion signal, but still exist that as shown in Fig. 4. Another explanation for the insensitivity of ASL to bandwidth selection is severely limited power to detect an effect because of the usually low SNR of the ASL signal. To gain power, we developed a linear mixed effect model that separated out random variations within subjects and fixed effects from changing bandwidths and added a co-factor to account for root mean square (RMS) noise variations between bandwidth settings. This approach still did not reveal significant N2 ghosting at the location of ROI3 inside of brain and only variations in ROI2 that is dominant to contributions of N2 ghosting were significant effect by bandwidth. Yet another, perhaps most likely explanation for lack of significant findings with ROI3 may be that the ROIs were too large and heterogeneous and effects "washed-out," because voxel-based analysis obtained significant results. To the best of our knowledge, no previous studies have examined the effects of different EPI readout bandwidths on an ASL perfusion MRI measurement.

The amplitude of $\mathrm{N} 2$ ghosts is depended on the selections of the bandwidth, but this dependence seems diminished in the brain. The first column of Table 1, listing values from ROI1 outside the brain, clearly shows that normalized noise is similar among the three bandwidth values. The second column of the table, listing values from ROI 2 that is still outside the brain but in a region potentially affected by N2 ghosting shows the N2 ghost amplitude decreasing with bandwidth values similarly to what was obtained in the phantom study (Fig. 1). A factor 2 in absolute N2 ghosting amplitude is found between the $2.442 \mathrm{kHz} /$ pixel and the $2.790 \mathrm{kHz} /$ pixel $(1.198$ 
Table 3 List of the EPI bandwidths used on MRI studies of human brain using arterial spin labeling (ASL), including the parameters of matrix, TE, bandwidth, field strength and ASL methods

\begin{tabular}{lllllll}
\hline List & Reference & Matrix & TE $(\mathrm{ms})$ & Bandwidth (KHz/pixel) & Field strength (T, Tesla) & ASL methods \\
\hline 1 & Francis et al. [19] & $64 \times 64$ & 24.0 & 1.50 & 3.0 & LL-FAIR \\
2 & Wang et al. [20] & $64 \times 64$ & 17.0 & 3.00 & 3T_Trio & 1.5T_Vision \\
3 & Gunther et al. [21] & $64 \times 64$ & 17.7 & 1.25 & 3T_Bruker & ITS-FAIR \\
4 & Mildner et al. [22] & $64 \times 64$ & 45.0 & 1.56 & 3T_Bruker & 3T_Biospec \\
5 & Trampel et al. [23] & $64 \times 64$ & 22.0 & 1.56 & 1.5T_Signa & CASL \\
6 & Luh et al. [24] & $64 \times 64$ & NM & 1.95 & 3T_Signa & PICORE \\
7 & Pollock et al. [25] & $48 \times 64$ & 28 & 1.3 & 3T_Allegra & 1.5T_Symphony \\
8 & Pollock et al. [25] & $48 \times 64$ & 28 & 1.3 & 3T_Trio & 3T_Trio \\
9 & Abler et al. [26] & $64 \times 64$ & 17 & 3.00 & 3T_MedSpec & 1.5T_Vision \\
10 & Noguchi et al. [27] & $64 \times 64$ & 26 & 1.53 & 1.5T_Intera & PICORE \\
11 & Qiu et al. [28] & $64 \times 64$ & 26 & 2.05 & 1.5T_Signa & CASL \\
12 & Wang et al. [29] & $64 \times 64$ & 17 & 3.0 & 3T_Trio & CASL \\
13 & Moller et al. [30] & $64 \times 64$ & 50 & 1.56 & 1.5T_Sonata & 1.4T_Sonata \\
14 & Warmuth et al. [31] & $80 \times 128$ & 22 & 1.25 & CASL \\
15 & Hermes et al. [32] & $63 \times 64$ & 42 & 1.22 & 3T_Inova & PAIR/PICORE \\
16 & Biagi et al. [33] & $40 \times 64$ & 22 & 1.56 & 2.60 & PICORE \\
17 & DA Holm et al. [34] & $64 \times 64$ & 20 & 3.00 & PICORE \\
18 & Campbell et al. [35] & $64 \times 64$ & 15 & 3.00 & 3.12 &
\end{tabular}

Bandwidth values varied depending on the gradient system of the MRI instrument but they are likely independent from the ASL methods used. None of the studies in this table used a bandwidth within a bad Nyquist (N2) ghost regime suggested by Fig. 1b. In generally, it is recommended to use a high bandwidth value that speeds up data acquisition and may also reduce N2 ghosting, according to the graph in Fig. $1 \mathrm{~b}$

$T E$ echo time, $N M$ not mentioned, $L L$ Look-Locker, FAIR flow-sensitive alternating inversion-recovery, PICORE proximal inversion with correction for off-resonance effects, ITS-FAIR inflow turbo sampling FAIR, CASL continuous arterial spin labeling

vs. 0.468). Finally, the third column of the table, listing values from ROI 3 inside the brain represents the amplitude of the image plus the $\mathrm{N} 2$ ghosting overlay. No difference in signal variation is found for ROI3, which could be related again to select the large size of the ROI in brain area or due to poor SNR.

The phantom study shows, as expected, that the magnitude of the N2 ghost varies as a function of the EPI readout-encoding bandwidth, as the gradient vibrations pass through the mechanical resonance condition of the MR gradient system. Using the matrix size of $64 \times 64$, the magnitude of the $\mathrm{N} 2$ ghost initially increased at $2.442 \mathrm{kHz} /$ pixel bandwidth and then decreased after $2.60 \mathrm{kHz} /$ pixel bandwidth shown in Fig. 1b. One obvious solution to reducing N2 ghosting is an increase of the EPI readout-encoding bandwidth within the limitation of the gradient performance, but the signal can be fluctuated at a certain bandwidth. At higher magnetic fields, increasing the bandwidth also decreases the vulnerability of EPI to susceptibility distortions in regions with inhomogeneous fields [16]. Similarly, increasing echo spacing decreases sensitivity because of the transverse relaxation of the signal, while a large increase of bandwidth could become prohibitive because oscillating gradients can induce peripheral nerve stimulation. Together, the range of variations of EPI readout bandwidth and equivalently echo spacing can be too limited at high magnetic fields to effectively eliminate N2 ghosting.
Although functional studies are usually performed using a BOLD-based sequence, ASL-based functional studies have gained increasing interest for a variety of reasons, including increased sensitivity to extremely slow frequency stimuli [17]. The additional advantage of ASL-based functional study is that we can simultaneously obtain functional activation maps of blood flow by subtracting the labeled image from the control image as well as of BOLD by adding the two images [18]. N2 ghosting might still exist and BOLDbased activation may be compromised if the bandwidth is not optimized to minimize N2 ghosting. In our phantom and human brain studies, the noise of the raw images, both control and labeled images, was enhanced for certain bandwidth values. To find out what other groups used for bandwidth, we searched the PubMed database with the keyword of "arterial spin labeling MRI" and found about 432 papers. Of those only 19 papers listed values for EPI bandwidth [19-37]. A summary of findings from all the reviewed studies is presented in Table 3 together with echo time, the EPI bandwidth, magnetic field strength, and the type of ASL methods. Bandwidth values varied depending on the gradient system of the MR instrument but they are likely independent from the ASL methods used. None of the studies in the Table 3 used a bandwidth within a bad N2 ghost regime suggested by Fig. $1 \mathrm{~b}$. In generally, it is recommended to use a high bandwidth value that speeds up data acquisition and may also reduce N2 ghosting, according to the graph in Fig. $1 \mathrm{~b}$. 
We did not perform fMRI experiments in this study. Future studies with ASL-MRI should investigate implications of bandwidth selection for fMRI to demonstrate the Nyquist ghost effect on fMRI measurements. Previous fMRI studies demonstrated that variable degrees of scanner noise, including variations in EPI bandwidth, affect activation patterns in fMRI [38-43]. In addition, previous investigations showed that N2 ghosting can affect measurements of diffusion anisotropy such as fractional anisotropy and the relative anisotropy whereas mean diffusivity was not affected $[12,13]$. The result of this study also showed that one should stay away from mechanical vibrations, which can result in artificial activation patterns in BOLD-based and ASL-based functional MRI studies [44].

This study has several limitations: one limitation is that we did not account for our statistics loss of signal-to-noise due to N2 ghosting. Since signal averaging is essential in ASL, it is conceivable that $\mathrm{N} 2$ ghosting reduces the coherence of the ASL signal between subsequent scans and thus diminishing efficiency of signal averaging to gain SNR. Lack to account for reduced SNR may have selectively reduced our power to detect effects of N2 ghosting. Another limitation is that the data in this study were acquired using various bandwidths, but keeping the TE constant. Although this would control for changes in $\mathrm{T} 2 *$ weighting, a different choice of bandwidth in practice should lead to different choices of TE. Since a change of TE could have potentially led to different levels of Nyquist ghost (e.g. due to decaying eddy currents sampled therefore at different time points), the results with fixed TE cannot be extended necessarily to other cases. However, in general, an ASL acquisition with a gradient-echo EPI sequence is usually performed with a minimum echo time to increase SNR in raw images, making N2 ghosting again mainly depended on the selection of EPI bandwidth rather than on the selection of TE. Lastly another limitation is that we did not distinguish artifacts caused by the $\mathrm{N} 2$ ghost noise from those caused by B0 inhomogeneity. Moreover, we did not investigate $\mathrm{N} 2$ ghosting effects for multi-channel receivers that could potential amplify the effect.

\section{Conclusion}

The major finding of this study is that N2 ghosting can have a significant effect on the ASL signal. Based on the findings of voxel-wise analysis in PWI which showed that N2 ghosts had influence in the area of overlaps, quantitative CBF measurements that require extra EPI scans to determine the equilibrium state signal should be cautious. Therefore, careful tuning of the bandwidth of EPI is necessary to avoid artifacts in the ASL signal from N2-ghosting.

Acknowledgments The authors thank Miss Sun-Hee Lee for technical assistance and also thank Dr. Jiongjiong Wang at the Department of
Radiology of University of Pennsylvania Medical Center in Philadelphia for providing the FAIR-ASL sequence. The authors are grateful to Dr. Michael W. Weiner at the Center for Imaging of Neurodegenerative Disease of University of California-San Francisco for invaluable support for this work. This research was supported by the Kyung Hee University Research Fund in 2006 (KHU-20061234).

Open Access This article is distributed under the terms of the Creative Commons Attribution Noncommercial License which permits any noncommercial use, distribution, and reproduction in any medium, provided the original author(s) and source are credited.

\section{References}

1. Tomasi DG, Ernst T (2003) Echo planar imaging at 4 Tesla with minimum acoustic noise. J Magn Reson Imaging 18(1):128-130

2. Yao GZ, Mechefske CK, Rutt BK (2004) Characterization of vibration and acoustic noise in a gradient-coil insert. Magn Reson Mater Phy 17(1):12-27

3. Price DL, De Wilde JP, Papadaki AM, Curran JS, Kitney RI (2001) Investigation of acoustic noise on 15 MRI scanners from 0.2 T to 3 T. J Magn Reson Imaging 13(2):288-293

4. Weiskopf N, Hutton C, Josephs O, Turner R, Deichmann R (2007) Optimized EPI for fMRI studies of the orbitofrontal cortex: compensation of susceptibility-induced gradients in the readout direction. Magn Reson Mater Phys 20(1):39-49

5. Schmithorst VJ, Dardzinski BJ, Holland SK (2001) Simultaneous correction of ghost and geometric distortion artifacts in EPI using a multiecho reference scan. IEEE Trans Med Imaging 20(6):535539

6. Hu X, Le TH (1996) Artifact reduction in EPI with phase-encoded reference scan. Magn Reson Med 36(1):166-171

7. Neufeld A, Assaf Y, Graif M, Hendler T, Navon G (2005) Susceptibility-matched envelope for the correction of EPI artifacts. Magn Reson Imaging 23(9):947-951

8. Williams DS, Detre JA, Leigh JS, Koretsky AP (1992) Magnetic resonance imaging of perfusion using spin inversion of arterial water. Proc Natl Acad Sci USA 89(1):212-216

9. Jahng GH, Zhu XP, Matson GB, Weiner MW, Schuff N (2003) Improved perfusion-weighted MRI by a novel double inversion with proximal labeling of both tagged and control acquisitions. Magn Reson Med 49(2):307-314

10. Kim SG (1995) Quantification of relative cerebral blood flow change by flow-sensitive alternating inversion recovery (FAIR) technique: application to functional mapping. Magn Reson Med 34(3):293-301

11. Buxton RB, Frank LR, Wong EC, Siewert B, Warach S, Edelman RR (1998) A general kinetic model for quantitative perfusion imaging with arterial spin labeling. Magn Reson Med 40(3):383-396

12. Jahng GH, Weiner MW, Schuff N (2008) Diffusion anisotropy indexes are sensitive to selecting the EPI readout-encoding bandwidth at high-field MRI. Magn Reson Imaging 26(5):676-682

13. Porter DA, Calamante F, Gadian DG, Connelly A (1999) The effect of residual Nyquist ghost in quantitative echo-planar diffusion imaging. Magn Reson Med 42(2):385-392

14. Luh WM, Wong EC, Bandettini PA, Hyde JS (1999) QUIPSS II with thin-slice TI1 periodic saturation: a method for improving accuracy of quantitative perfusion imaging using pulsed arterial spin labeling. Magn Reson Med 41(6):1246-1254

15. Kim SG, Hu X, Ugurbil K (1994) Accurate T1 determination from inversion recovery images: application to human brain at 4 Tesla. Magn Reson Med 31(4):445-449 
16. Mechefske CK, Geris R, Gati JS, Rutt BK (2002) Acoustic noise reduction in a $4 \mathrm{~T}$ MRI scanner. Magn Reson Mater Phys 13(3):172-176

17. Wang J, Aguirre GK, Kimberg DY, Roc AC, Li L, Detre JA (2003) Arterial spin labeling perfusion fMRI with very low task frequency. Magn Reson Med 49(5):796-802

18. Wong EC, Buxton RB, Frank LR (1997) Implementation of quantitative perfusion imaging techniques for functional brain mapping using pulsed arterial spin labeling. NMR Biomed 10(4-5):237-249

19. Francis ST, Bowtell R, Gowland PA (2008) Modeling and optimization of Look-Locker spin labeling for measuring perfusion and transit time changes in activation studies taking into account arterial blood volume. Magn Reson Med 59(2):316-325

20. Wang Z, Wang J, Connick TJ, Wetmore GS, Detre JA (2005) Continuous ASL (CASL) perfusion MRI with an array coil and parallel imaging at 3T. Magn Reson Med 54(3):732-737

21. Gunther M, Bock M, Schad LR (2001) Arterial spin labeling in combination with a Look-Locker sampling strategy: inflow turbosampling EPI-FAIR (ITS-FAIR). Magn Reson Med 46(5):974-984

22. Mildner T, Trampel R, Moller HE, Schafer A, Wiggins CJ, Norris DG (2003) Functional perfusion imaging using continuous arterial spin labeling with separate labeling and imaging coils at 3 T. Magn Reson Med 49(5):791-795

23. Trampel R, Mildner T, Goerke U, Schaefer A, Driesel W, Norris DG (2002) Continuous arterial spin labeling using a local magnetic field gradient coil. Magn Reson Med 48(3):543-546

24. Luh WM, Wong EC, Bandettini PA, Ward BD, Hyde JS (2000) Comparison of simultaneously measured perfusion and BOLD signal increases during brain activation with T(1)-based tissue identification. Magn Reson Med 44(1):137-143

25. Pollock JM, Whitlow CT, Deibler AR, Tan H, Burdette JH, Kraft RA, Maldjian JA (2008) Anoxic injury-associated cerebral hyperperfusion identified with arterial spin-labeled MR imaging. AJNR Am J Neuroradiol 29(7):1302-1307

26. Abler B, Hofer C, Viviani R (2008) Habitual emotion regulation strategies and baseline brain perfusion. Neuroreport 19(1):21-24

27. Noguchi T, Yoshiura T, Hiwatashi A, Togao O, Yamashita K, Nagao E, Shono T, Mizoguchi M, Nagata S, Sasaki T, Suzuki SO, Iwaki T, Kobayashi K, Mihara F, Honda H (2008) Perfusion imaging of brain tumors using arterial spin-labeling: correlation with histopathologic vascular density. AJNR Am J Neuroradiol 29(4):688-693

28. Qiu M, Ramani R, Swetye M, Constable RT (2008) Spatial nonuniformity of the resting CBF and BOLD responses to sevoflurane: in vivo study of normal human subjects with magnetic resonance imaging. Hum Brain Mapp 29(12):1390-1399

29. Wang Z, Aguirre GK, Rao H, Wang J, Fernandez-Seara MA, Childress AR, Detre JA (2008) Empirical optimization of ASL data analysis using an ASL data processing toolbox: ASLtbx. Magn Reson Imaging 26(2):261-269

30. Moller HE, Mildner T, Preul C, Zimmer C, Cramon DY (2007) Assessment of collateral supply by two-coil continuous arterial spin labeling after coil occlusion of the internal carotid artery. AJNR Am J Neuroradiol 28(7):1304-1305
31. von Warmuth $\mathrm{C}$, Nagel S, Hegemann O, Wlodarczyk W, Ludemann L (2007) Accuracy of blood flow values determined by arterial spin labeling: a validation study in isolated porcine kidneys. J Magn Reson Imaging 26(2):353-358

32. Hermes M, Hagemann D, Britz P, Lieser S, Rock J, Naumann E, Walter C (2007) Reproducibility of continuous arterial spin labeling perfusion MRI after 7 weeks. Magn Reson Mater Phys 20(2):103-115

33. Biagi L, Abbruzzese A, Bianchi MC, Alsop DC, Del Guerra A, Tosetti M (2007) Age dependence of cerebral perfusion assessed by magnetic resonance continuous arterial spin labeling. J Magn Reson Imaging 25(4):696-702

34. Holm DA, Sidaros K (2006) Slice profile optimization in arterial spin labeling using presaturation and optimized RF pulses. Magn Reson Imaging 24(9):1229-1240

35. Campbell AM, Beaulieu C (2006) Comparison of multislice and single-slice acquisitions for pulsed arterial spin labeling measurements of cerebral perfusion. Magn Reson Imaging 24(7):869-876

36. Federspiel A, Muller TJ, Horn H, Kiefer C, Strik WK (2006) Comparison of spatial and temporal pattern for fMRI obtained with BOLD and arterial spin labeling. J Neural Transm 113(10):14031415

37. Figueiredo PM, Clare S, Jezzard P (2005) Quantitative perfusion measurements using pulsed arterial spin labeling: effects of large region-of-interest analysis. J Magn Reson Imaging 21(6):676-682

38. Gaab N, Gabrieli JD, Glover GH (2007) Assessing the influence of scanner background noise on auditory processing. II. An fMRI study comparing auditory processing in the absence and presence of recorded scanner noise using a sparse design. Hum Brain Mapp 28(8):721-732

39. Gaab N, Gabrieli JD, Glover GH (2007) Assessing the influence of scanner background noise on auditory processing. I. An fMRI study comparing three experimental designs with varying degrees of scanner noise. Hum Brain Mapp 28(8):703-720

40. Bartsch AJ, Homola G, Thesen S, Sahmer P, Keim R, Beckmann CF, Biller A, Knaus C, Bendszus M (2007) Scanning for the scanner: FMRI of audition by read-out omissions from echo-planar imaging. Neuroimage 35(1):234-243

41. Tamer G, Talavage TM, Luh WM, Ulmer JL (2004) Characterizing the amplitude and spatial extent of the cortical response in auditory cortex to acoustic scanner noise generated during echo-planar image acquisition in functional magnetic resonance imaging. Conf Proc IEEE Eng Med Biol Soc 3:1899-1902

42. Tamer G, Talavage TM, Ulmer JL (2004) Characterizing the attenuation and/or saturation effect of the acoustic scanner noise in auditory event-related functional magnetic resonance imaging. Conf Proc IEEE Eng Med Biol Soc 3:1868-1871

43. Zhang N, Zhu XH, Chen W (2005) Influence of gradient acoustic noise on fMRI response in the human visual cortex. Magn Reson Med 54(2):258-263

44. Delakis I, Petala K, De Wilde JP (2005) MRI receiver frequency response as a contributor to Nyquist ghosting in echo planar imaging. J Magn Reson Imaging 22(2):324-328 